\title{
Electronic cigarettes: The potential risks outweigh the benefits
}

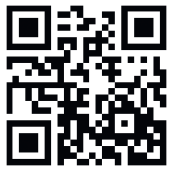

Should the legislation on the sale and promotion of electronic cigarettes (e-cigarettes) be similar to that for tobacco smoking, or are they different? Is there potential medical benefit in their availability and use? I shall argue that they should be regulated, based on the overwhelming potential for their harm to society, and particularly to those that they are intended to 'help'. Firstly, the evidence for their being an effective method for smoking cessation is unconvincing. Secondly, they are a means for maintaining nicotine addiction and dependence. Thirdly, they may even encourage more habitual use of nicotine, which, in time, might encourage a switch to cigarette smoking. Other concerns are that their safety has not been proven in large studies of long-term use, their effect during pregnancy is unknown, and that many e-cigarettes are owned, produced and aggressively promoted by the tobacco industry. The tobacco industry has a track record of scant concern for the fact that they are promoting the single most important preventable cause of malignancy worldwide, apart from their other effects. Thus, I will argue that this new 'vice' should be subjected to the same, if not more rigid, scrutiny applied to tobacco and habit-forming drugs before it is released freely to the unsuspecting population.

Arguably, the only sound justification for physicians to support the introduction of e-cigarettes would be if they proved an effective means of helping addicted tobacco smokers quit. However, even if they prove effective in this, it would have to be shown that they did not do harm by convincing new clients that smoking can be safe! Or, as intended by some of the manufacturers, acting as gateway devices to cigarette smoking.

The first priority in healthcare is safety: safety for the user and for the bystander. Primary concerns relate to the lack of regulation in manufacturing processes in some parts of the world. ${ }^{[1]}$ There are several hundred brands of e-cigarettes and certain studies have documented e-cigarettes contaminated with diethylene glycol, nitrosamines, acetaldehyde and acetone. ${ }^{[1]}$ Although, it is likely that more 'reputable' brands have stricter manufacturing controls and thus contain only what is written on the package insert, these too may not be safe. There are data showing acutely increased pulmonary resistance after smoking and there is a complete lack of long-term safety data. ${ }^{[2]}$ Furthermore, nicotine itself is not entirely safe: it is toxic at high doses, increases insulin resistance, is immunosuppressive and directly stimulates pulmonary mucus secretion. ${ }^{[3,4]}$ Tobacco smoking was considered to be safe and fashionable in the early 1900s, until Richard Doll's revolutionary work in the 1950s showed otherwise. ${ }^{[5]}$ Over 50 million tobacco-related deaths are estimated to have occurred in the past 10 years despite us knowing full well the harms of tobacco smoke. ${ }^{[6]}$

What of efficacy? Some convincing data exist that smokers of e-cigarettes smoke fewer tobacco cigarettes. ${ }^{[7]}$ There are scant data, however, on their efficacy as a bridge to stop smoking. ${ }^{[8]}$ To date, the results presented are from small poorly-designed studies with inadequate blinding and control/placebo arms, and have shown no clear evidence that e-cigarettes assist individuals in stopping smoking. The two largest studies, both from the same centre, evaluated 27 subjects completing a 6-month study (no control arm) and 125 in a 1-year study (unblinded). Thus to date, efficacy and safety are based on trial data from a few hundred patients alone. ${ }^{[7,8]}$

E-cigarettes are not necessarily cheaper alternatives to tobacco. If their use does not result in complete smoking cessation (tobacco and e-cigarettes) they may merely perpetuate the financial burden of smoking. If marketed as fashionable and progressive, like the hookah pipe ('hubbly-bubbly'), they may additionally yield a new generation of e-smokers, chemically addicted to nicotine. Nicotine, although not known to be a bridge to narcotic or other drug usage, potentially opens the door to chemical tolerance, and long-term users may seek increasingly higher nicotine concentrations, ultimately resorting to tobacco. E-cigarettes are advocated as a 'healthier alternative', but the obvious long-term addiction to nicotine remains unaddressed.

E-cigarettes have not been shown to be safe or effective in smoking cessation. They contain nicotine, which is toxic and addictive, and tobacco companies are selling them. How can they be good? The e-cigarette industry needs to be tightly regulated, and independent assessment of the harms needs to be made, or we risk replacing one evil for another. E-cigarettes may be less dangerous than tobacco, but given that tobacco kills $50 \%$ of its users, what would not be safer?

\section{R N van Zyl-Smit}

Division of Pulmonology and Lung Institute, University of Cape Town, South Africa

richard.vanzyl-smit@uct.ac.za

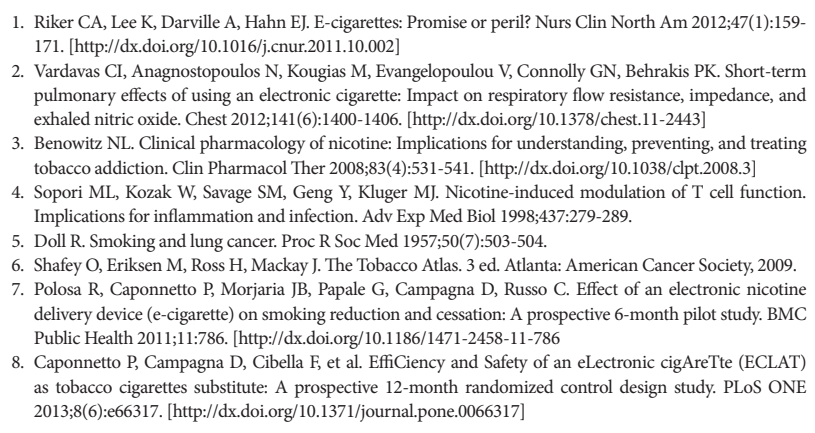

S Afr Med J 2013;103(11):833. DOI:10.7196/SAMJ.7435 